\title{
riccafd
}

Revista Iberoamericana de Ciencias de la Actividad Física y el Deporte

\section{ACCIONES TÉCNICO-TÁCTICAS, FACTORES CONTEXTUALES Y RENDIMIENTO DEPORTIVO DE LOS EQUIPOS DE CONCACAF DURANTE LA CLASIFICACIÓN A RUSIA 2018}

\section{TECHNICAL-TACTICAL ACTIONS, CONTEXTUAL FACTORS AND SPORT PERFORMANCE OF CONCACAF TEAMS DURING CLASSIFICATION TO RUSSIA 2018}

Ugalde-Ramírez, JA. ${ }^{1}$, Rivas-Borbón, OM. ${ }^{1}$, Rojas-Valverde, D. ${ }^{1}$

${ }^{1}$ Ugalde-Ramírez, JA. Escuela Ciencias del Movimiento Humano y Calidad de Vida, Universidad Nacional, Costa Rica. a.ugalde07@hotmail.com

${ }^{1}$ Rivas-Borbón, OM. Escuela Ciencias del Movimiento Humano y Calidad de Vida, Universidad Nacional, Costa Rica.miltonribo@gmail.com

${ }^{1}$ Rojas-Valverde, D. Escuela Ciencias del Movimiento Humano y Calidad de Vida, Universidad Nacional, Costa Rica.drojasv@hotmail.com

Código UNESCO: 5899. Otras Especialidades Pedagógicas (Educación Física y deporte)

Clasificación Consejo de Europa: 4. Educación Física y deporte comparado

Correspondencia: Ugalde-Ramírez, Jose Alexis a.ugalde07@hotmail.com

DOI: http://dx.doi.org/10.24310/riccafd.2019.v8i1.5764

\section{RESUMEN}

El objetivo de este estudio fue determinar cuáles acciones técnico-tácticas y factores contextuales se relacionaron con el rendimiento deportivo de las selecciones absolutas de CONCACAF durante la fase clasificatoria a la Copa Mundial de Rusia 2018. Se utilizaron las estadísticas de los 10 partidos jugados por cada una de los 6 equipos nacionales (30 partidos, 60 observaciones). Los resultados indican que las acciones técnico-tácticas que se relacionaron con el rendimiento y que diferenciaron a los equipos clasificados y no clasificados, así como a los equipos que obtenían victorias con los que empataban o perdían, fueron: los goles, remates a marco realizados, pases, y posesión de balón, asimismo estos equipos consiguieron mayor cantidad de victorias cuando jugaban de locales. En conclusión, las acciones técnico-tácticas relacionadas con el rendimiento coinciden con las reportadas en la literatura, además, el hecho de jugar de local representó una ventaja para los equipos.

Palabras clave: fútbol, indicadores, rendimiento, competición. 


\section{ABSTRACT}

The objective of this study was to determine which technical-tactical actions and contextual factors were related to the sports performance of the CONCACAF national teams during the qualifying phase of the 2018 World Cup Russia. The statistics of the 10 matches played by each of 6 national teams team were used (30 matches, 60 observations). The results indicate that the technical-tactical actions that were related to the sport performance and that differentiated the classified and unclassified teams, as well as the teams that obtained victories with the that tied or lost, were: the goals, shots on target, passes, and possession of the ball, also these teams got more victories when playing from home. In conclusion, the technical-tactical actions related to performance coincide with those reported in the literature, moreover, the fact of playing at home represented an advantage for the teams.

Key Words: soccer, indicators, performance, competition.

\section{INTRODUCCIÓN}

El futbol es un deporte en el cual se llevan a cabo numerosos estudios que analizan las tendencias de juego tanto ofensivas como defensivas desarrolladas por los diferentes equipos, selecciones nacionales y clubes en las ligas y torneos. Estas investigaciones hacen una descripción y valoración de diferentes acciones técnico-tácticas que los equipos ejecutan en la competencia y a su vez como estos elementos del juego influye sobre el resultado y rendimiento del equipo ${ }^{1,2}$.

Los parámetros técnico-tácticos que parecen tener mayor relación y relevancia para el desarrollo efectivo del juego, principalmente en su fase ofensiva son: el tiempo y el porcentaje de posesión de balón que posee un equipo, la cantidad de pases realizados y su efectividad, así como los remates realizados y goles anotados $^{3,4}$. Asimismo, de estos análisis, también se consideran acciones como los saques de esquina, faltas, tarjetas amarillas y rojas, fueras de juego, debido a que son acciones que suceden y se ejecutan por los equipos durante la dinámica de juego ${ }^{5,6}$.

En el contexto del fútbol competitivo se han identificado una serie de factores contextuales y situacionales que requieren atención como lo es la localía, la posición en la tabla, el momento del partido en que se consigue un gol, el nivel del oponente, así como el resultado del partido; pues podrían influir y generar que los equipos cambien sus modelos y estilos de juego $0^{7,8,9,10,11}$, de igual manera, pueden condicionar los comportamientos físicos, técnicos y tácticos durante los partidos $^{12,13 .}$

Gran parte de los estudios realizados en el fútbol para evaluar los comportamientos técnico-tácticos de los jugadores y equipos se han llevado a cabo con datos obtenidos de ligas y torneos europeos como por ejemplo la Liga españo- 
la ${ }^{5,14}$, la francesa ${ }^{15}$, la italiana ${ }^{16}$ y ligas inglesas ${ }^{17}$. Algunos otros estudios se han realizados con estadísticas de equipos de las ligas suramericanas como la liga brasileña ${ }^{8,18}$ y en menor cantidad, en competiciones asiáticas ${ }^{19}$.

Otros estudios fueron realizados con datos de los campeonatos mundiales de fútbol. En esta línea, encontramos estudios del Mundial Sudáfrica 2010, que compararon el rendimiento físico y técnico de selecciones ganadoras y perdedoras $^{20}$. Con datos de los mundiales del 2002, 2006 y 2010, Castellano, Casamichana y Lagos ${ }^{21}$ compararon las acciones técnicas realizadas por los equipos según el resultado obtenido en el partido. Chmura et al., $2017^{22}$ y Krol et al. ${ }^{23}$, analizaron los pases realizados por los equipos y jugadores en el mundial de Brasil 2014. Asimismo, otros trabajos han relacionado variables ofensivas y defensivas técnico-tácticas con el rendimiento deportivo de los equipos en los mundiales del 2010 y $2014^{3,6}$.

Sin embargo, no se conocen estudios que hayan analizado el rendimiento de selecciones nacionales que participaron en los procesos eliminatorios hacia un mundial en la Confederación, Centroamericana, Norte y Caribe de Fútbol (CONCACAF). Por tanto, el propósito de este estudio fue determinar cuáles acciones técnico-tácticas y factores contextuales se relacionaron con el rendimiento deportivo de las selecciones nacionales de CONCACAF durante la última fase del proceso clasificatorio para la Copa Mundial de Rusia 2018.

\section{MATERIALES Y MÉTODO}

Se tomaron en cuenta las selecciones de fútbol absolutas del área de CONCACAF (Estados Unidos, México, Honduras, Costa Rica, Panamá y Trinidad y Tobago) que participaron en la hexagonal final de la clasificatoria a la Copa Mundial de Rusia 2018. Cada selección disputó 10 partidos, de los cuales cinco partidos los realizaron en condición de locales y cinco de visitante. En total se analizaron 30 partidos y 60 observaciones para cada variable.

Los datos de cada uno de los partidos efectuados durante la hexagonal final clasificatoria, se obtuvieron accesando la página web oficial de la CONCACAF en el apartado resultado de competiciones ${ }^{24}$; procediendo a seleccionar cada uno de los partidos disputados por las selecciones para obtener las estadísticas de las acciones técnico-tácticas realizadas por cada equipo en cada uno de los partidos.

La confiabilidad de los datos proporcionados por el sitio web fue valorada, comparándolos con los obtenidos del análisis de tres partidos seleccionados aleatoriamente de la eliminatoria por parte de tres evaluadores externos a la investigación. Pruebas $t$-student para una muestra, no evidenciaron diferencias entre ambos registros $(p>0.05)$.

Los factores contextuales considerados fueron el lugar donde se jugó el partido (local o visita) y el resultado del partido (ganó, empató o perdió).

\footnotetext{
29 ACCIONES TÉCNICO-TÁCTICAS, FACTORES CONTEXTUALES Y RENDIMIENTO DEPORTIVO DE LOS EQUIPOS DE CONCACAF DURANTE LA CLASIFICACIÓN A RUSIA 2018
} 
Las acciones técnico-tácticas se clasificaron en acciones defensivas y acciones ofensivas. Las acciones ofensivas fueron: goles anotados, remates a marco realizados, tiros de esquina efectuados, faltas recibidas, fueras de juego recibidos (se toman como ofensivos puesto que se generan en la fase de ataque del equipo), pases realizados, efectividad de pases y porcentaje de posesión de balón. Por otro lado, las acciones defensivas fueron: goles recibidos, remates a marco recibidos, tiros de esquina defendidos, faltas realizadas, fueras de juego provocados (se toman como defensivos puesto que se generan en una acción defensiva que deja a los jugadores rivales inhabilitados para jugar), tarjetas amarillas y tarjetas rojas.

Se consideró el rendimiento de cada equipo en dos variables, una categórica que dividió a los equipos en clasificados y no clasificados al mundial; y la otra continua; que fue la cantidad de puntos obtenidos por cada equipo en la hexagonal.

\section{Análisis estadístico}

Se obtuvieron valores promedio y desviaciones estándar para cada variable. Se realizaron pruebas de Shapiro-Wilk para la normalidad de los datos, obteniendo resultados $p<0,05$. Por tanto, se realizaron pruebas no paramétricas. Para comparar las variables técnico-tácticas de los equipos que clasificaron y equipos que no clasificaron al mundial, asimismo para comparar las variables según el lugar donde jugaba el equipo (local o visita) se aplicaron pruebas U Mann-Whitney $(Z)$. Se evaluó el tamaño del efecto mediante la $R^{2}$ calculada $(Z / \sqrt{ } n)^{25}$ y cualificada como: $R^{2}=0,1$ (bajo), $R^{2}=0,3$ (medio), $R^{2}=0,5$ (grande), $R^{2}=0,7$ (muy grande) ${ }^{26}$.

Para comparar los indicadores técnico-tácticos según los resultados obtenidos por el equipo en los partidos (ganó, empató o perdió) se aplicaron pruebas Kruskal-Wallis $(H)$. Para los casos que requirió determinar entre cuales condiciones se encontraban las diferencias, se realizaron los análisis post hoc siguiendo lo indicado por O'Donogue ${ }^{27}$. Se obtuvo el tamaño del efecto por medio del EpsiIon cuadrado $\left(\varepsilon^{2}\right)$ y cualificado como: $\varepsilon^{2}=0,1$ (pequeño), $\varepsilon^{2}=0,3$ ( moderado) $\varepsilon^{2}=0,5$ (grande) $)^{28}$.

Se realizaron correlaciones de Spearman para determinar las relaciones entre los indicadores técnico-tácticos y el rendimiento de los equipos expresado en la cantidad de puntos conseguidos.

Para todos los análisis se consideró un nivel de significancia $p<0,05$. Los mismos fueron realizados en el Paquete Estadístico para Ciencias Sociales (SPSS) (IBM, SPSS, V.23, USA).

\section{RESULTADOS}

Los resultados de este estudio muestran en la tabla 1 diferencias entre los equipos que clasificaron y los que no clasificaron al mundial en algunos indicadores técnico-tácticos. Específicamente, los equipos que clasificaron recibieron 
menor cantidad de goles, realizaron mayor cantidad de pases, presentaron mayor porcentaje de posesión de balón, recibieron más cantidad de faltas e incurrieron más veces en fueras de fuego que los equipos que no clasificaron por partido.

Tabla 1. Acciones técnico-tácticas realizadas por partido por los equipos que clasificaron y que no clasificaron durante la fase clasificatoria.

\begin{tabular}{|c|c|c|c|c|c|}
\hline & Clasificados & No clasificados & $\mathbf{z}$ & $p$ & $T E\left(R^{2}\right)$ \\
\hline Acciones ofensivas & $\mathrm{n}=\mathbf{3 0}$ & $\mathrm{n}=\mathbf{3 0}$ & & & \\
\hline Goles anotados (c/p) & $1,30 \pm 1,08$ & $1,23 \pm 1,35$ & 0,658 & 0,511 & $0,08^{*}$ \\
\hline Remates a marco realizados $(c / p)$ & $4,53 \pm 2,60$ & $3,93 \pm 2,42$ & $-1,540$ & 0,123 & $0,2^{*}$ \\
\hline Tiros de esquina a favor (c/p) & $4,17 \pm 2,58$ & $3,97 \pm 1,77$ & $-0,278$ & 0,781 & $0,03^{\ddagger}$ \\
\hline Fuera de juego recibidos (c/p) & $1,57 \pm 1,13$ & $1,03 \pm 1,37$ & $-2,343$ & $0,019 *$ & $0,3^{\ddagger}$ \\
\hline Faltas recibidas $(c / p)$ & $13,33 \pm 4,52$ & $10,83 \pm 4,77$ & $-2,134$ & $0,033^{*}$ & $0,28^{\ddagger}$ \\
\hline Posesión de balón (\%) & $53,10 \pm 9,41$ & $46,87 \pm 9,20$ & $-2,258$ & $0,024^{*}$ & $0,29^{\ddagger}$ \\
\hline Pases correctos (c/p) & $246,77 \pm 74,15$ & $200,83 \pm 71,14$ & $-2,055$ & $0,040^{*}$ & $0,67^{z}$ \\
\hline Efectividad pases (\%) & $81,70 \pm 8,24$ & $80,77 \pm 8,00$ & $-0,467$ & 0,641 & $0,06^{*}$ \\
\hline \multicolumn{6}{|l|}{ Acciones defensivas } \\
\hline Goles recibidos (c/p) & $0,87 \pm 0,97$ & $1,70 \pm 1,29$ & $-2,939$ & $0,003^{*}$ & $0,38^{\ddagger}$ \\
\hline Remates a marco recibidos (c/p) & $3,77 \pm 2,43$ & $4,70 \pm 2,54$ & $-1,287$ & 0,198 & $0,16^{*}$ \\
\hline Tiros de esquina en contra $(c / p)$ & $4,17 \pm 2,18$ & $3,97 \pm 2,25$ & $-0,158$ & 0,875 & $0,02^{*}$ \\
\hline Fuera de juego provocados $(c / p)$ & $1,20 \pm 1,44$ & $1,40 \pm 1,10$ & $-1,268$ & 0,205 & $0,16^{*}$ \\
\hline Faltas cometidas (c/p) & $13,03 \pm 4,50$ & $11,13 \pm 4,93$ & $-1,541$ & 0,123 & $0,2^{¥}$ \\
\hline Tarjetas amarillas (c/p) & $1,63 \pm 1,18$ & $1,80 \pm 1,34$ & $-0,566$ & 0,571 & $0,07^{*}$ \\
\hline Tarjetas rojas (c/p) & $0,07 \pm 0,25$ & $0,13 \pm 0,34$ & $-0,853$ & 0,393 & $0,11^{*}$ \\
\hline
\end{tabular}

${ }^{*} p<0,05$.

$T E\left(R^{2}\right)$ tamaño del efecto *bajo, ${ }^{\ddagger}$ moderado, ${ }^{z}$ grande

$\mathrm{n}=$ cantidad de observaciones, $\mathrm{c} / \mathrm{p}=$ cantidad por partido, \%=porcentaje

Al comparar los resultados según el lugar donde se disputó el partido, se muestra en la tabla 2, que por partido los equipos que jugaban como locales anotaban más goles y realizaban más remates a marco y presentaban un mayor porcentaje de posesión de balón que los equipos visitantes. También, se observa que los jugadores de los equipos visitantes eran amonestados con tarjetas amarrillas más veces que los jugadores de los equipos locales.

Considerando el resultado obtenido en el partido, en la tabla 3, se muestra que los equipos ganadores presentan mayor cantidad de goles y remates a marco por partido que los equipos que empataban o perdían.

En la tabla 4, se observa que los equipos consiguieron mayor cantidad de victorias y empates (vistos como resultados positivos) cuando jugaban en localía que cuando lo hacían de visita. Los equipos que clasificaron no perdieron partidos cuando jugaron de locales. Por el contrario, los equipos que no clasificaron tuvieron más derrotas jugando de visita. 
Rev.Ib.CC. Act. Fís. Dep. 2019; 8(1): 27-38

Tabla 2. Acciones técnico-tácticas realizadas por partido por los equipos según el lugar donde se jugó el partido durante la fase clasificatoria.

\begin{tabular}{|c|c|c|c|c|c|}
\hline & Local & Visita & z & $p$ & $T E\left(R^{2}\right)$ \\
\hline Acciones ofensivas & $n=30$ & $\mathrm{n}=\mathbf{3 0}$ & & & \\
\hline Goles anotados (c/p) & $1,70 \pm 1,44$ & $0,83 \pm 0,74$ & $-2,476$ & $0,013 *$ & $0,32^{\ddagger}$ \\
\hline Remates a marco realizados $(\mathrm{c} / \mathrm{p})$ & $5,10 \pm 2,78$ & $3,37 \pm 1,88$ & $-2,408$ & $0,016^{*}$ & $0,31^{*}$ \\
\hline Tiros de esquina a favor $(c / p)$ & $4,53 \pm 2,52$ & $3,60 \pm 1,73$ & $-1,238$ & 0,216 & $0,16^{*}$ \\
\hline Fuera de juego recibidos (c/p) & $1,30 \pm 1,30$ & $1,30 \pm 1,26$ & $-0,062$ & 0,951 & $0,01^{*}$ \\
\hline Faltas recibidas $(c / p)$ & $12,80 \pm 4,62$ & $11,37 \pm 4,90$ & $-1,067$ & 0,286 & $0,14^{*}$ \\
\hline Posesión de balón (\%) & $52,23 \pm 9,95$ & $47,73 \pm 9,15$ & $-2,176$ & $0,030 *$ & $0,28^{\ddagger}$ \\
\hline Pases correctos (c/p) & $242,47 \pm 72,54$ & $205,13 \pm 75,28$ & $-1,878$ & 0,060 & $0,24^{*}$ \\
\hline Efectividad pases (\%) & $82,83 \pm 5,14$ & $79,63 \pm 10,04$ & $-0,896$ & 0,370 & $0,12^{*}$ \\
\hline \multicolumn{6}{|l|}{ Acciones defensivas } \\
\hline Goles recibidos (c/p) & $0,83 \pm 0,74$ & $1,73 \pm 1,41$ & $-2,668$ & $0,008^{*}$ & $0,34^{\ddagger}$ \\
\hline Remates a marco recibidos $(c / p)$ & $3,37 \pm 1,88$ & $5,10 \pm 2,78$ & $-2,408$ & $0,016^{*}$ & $0,31^{*}$ \\
\hline Tiros de esquina en contra (c/p) & $3,60 \pm 1,73$ & $4,53 \pm 2,52$ & $-1,238$ & 0,216 & $0,15^{*}$ \\
\hline Fuera de juego provocados (c/p) & $1,30 \pm 1,26$ & $1,30 \pm 1,30$ & $-0,062$ & 0,951 & $0,01^{*}$ \\
\hline Faltas cometidas (c/p) & $11,37 \pm 4,90$ & $12,80 \pm 4,62$ & $-1,067$ & 0,286 & $0,13^{*}$ \\
\hline Tarjetas amarillas (c/p) & $1,13 \pm 1,04$ & $2,20 \pm 1,29$ & $-2,855$ & $0,004 *$ & $0,37^{*}$ \\
\hline Tarjetas rojas $(\mathrm{c} / \mathrm{p})$ & $0,13 \pm 0,34$ & $0,07 \pm 0,25$ & $-0,853$ & 0,393 & $0,11^{*}$ \\
\hline
\end{tabular}

${ }^{*} p<0,05$,

$T E\left(R^{2}\right)$ tamaño del efecto *bajo, ${ }^{\ddagger}$ moderado, ${ }^{z}$ grande

$\mathrm{n}=$ cantidad de observaciones, $\mathrm{c} / \mathrm{p}=$ cantidad por partido, \%=porcentaje.

Tabla 3. Acciones técnico-tácticas realizadas por partido por los equipos según el resultado obtenido en los partidos durante la fase clasificatoria.

\begin{tabular}{|c|c|c|c|c|c|c|}
\hline & Ganó & Empató & Perdió & $H$ & $p$ & $T E\left(\varepsilon^{2}\right)$ \\
\hline Acciones ofensivas & $\mathrm{n}=\mathbf{2 1}$ & $n=18$ & $n=21$ & & & \\
\hline Goles anotados $(\mathrm{c} / \mathrm{p})$ & $2,43 \pm 1,20^{\mathrm{a}}$ & $0,89 \pm 0,58^{b}$ & $0,43 \pm 0,59^{b}$ & 34,361 & $0,000 *$ & $0,58^{\ddagger}$ \\
\hline Remates a marco realizados $(c / p)$ & $5,76 \pm 2,62^{\mathrm{a}}$ & $3,67 \pm 2,24^{b}$ & $3,19 \pm 1,88^{b}$ & 12,315 & $0,002 *$ & $0,21^{*}$ \\
\hline Tiros de esquina a favor $(c / p)$ & $3,48 \pm 2,06$ & $4,72 \pm 2,65$ & $4,10 \pm 1,81$ & 1,765 & 0,414 & $0,03^{*}$ \\
\hline Fuera de juego recibidos (c/p) & $1,05 \pm 0,86$ & $1,56 \pm 1,33$ & $1,33 \pm 1,56$ & 1,315 & 0,518 & $0,02^{*}$ \\
\hline Faltas recibidas (c/p) & $11,10 \pm 4,53$ & $14,11 \pm 4,44$ & $11,33 \pm 4,97$ & 4,978 & 0,083 & $0,08^{*}$ \\
\hline Posesión de balón (\%) & $51,48 \pm 9,41$ & $50,50 \pm 11,27$ & $47,05 \pm 8,24$ & 3,406 & 0,182 & $0,06^{*}$ \\
\hline Pases correctos $(c / p)$ & $256,05 \pm 65,99$ & $196,39 \pm 88,36$ & $215,05 \pm 63,68$ & 5,233 & 0,076 & $0,09^{*}$ \\
\hline Efectividad pases (\%) & $81,76 \pm 9,23$ & $80,28 \pm 7,63$ & $81,52 \pm 7,50$ & 0,830 & 0,660 & $0,01^{*}$ \\
\hline \multicolumn{7}{|l|}{ Acciones defensivas } \\
\hline Goles recibidos (c/p) & $0,43 \pm 0,59^{b}$ & $0,89 \pm 0,58^{b}$ & $2,43 \pm 1,20^{\mathrm{a}}$ & 33,591 & $0,000 *$ & $0,57^{*}$ \\
\hline Remates a marco recibidos $(c / p)$ & $3,19 \pm 1,88^{b}$ & $3,67 \pm 2,24^{b}$ & $5,76 \pm 2,62^{\mathrm{a}}$ & 12,315 & $0,002 *$ & $0,21^{*}$ \\
\hline Tiros de esquina en contra (c/p) & $4,10 \pm 1,81$ & $4,72 \pm 2,65$ & $3,48 \pm 2,06$ & 1,765 & 0,414 & $0,03^{*}$ \\
\hline Fuera de juego provocados (c/p) & $1,33 \pm 1,56$ & $1,56 \pm 1,33$ & $1,05 \pm 0,86$ & 1,315 & 0,518 & $0,02^{*}$ \\
\hline Faltas cometidas $(c / p)$ & $11,33 \pm 4,97$ & $14,11 \pm 4,44$ & $11,10 \pm 4,53$ & 4,978 & 0,083 & $0,08^{*}$ \\
\hline Tarjetas amarillas $(\mathrm{c} / \mathrm{p})$ & $1,43 \pm 1,35$ & $1,83 \pm 1,29$ & $1,90 \pm 1,09$ & 2,645 & 0,266 & $0,04^{*}$ \\
\hline Tarjetas rojas (c/p) & $0,14 \pm 0,35$ & $0,11 \pm 0,32$ & $0,05 \pm 0,21$ & 1,075 & 0,584 & $0,02^{*}$ \\
\hline
\end{tabular}

${ }^{*} p<0,05$, Letras distintas indican las diferencias.

$T E\left(\varepsilon^{2}\right)$ tamaño del efecto ${ }^{\ddagger}$ pequeño, ${ }^{\ddagger}$ moderado, , ${ }^{z}$ grande

$\mathrm{n}=$ cantidad de observaciones, $\mathrm{c} / \mathrm{p}=$ cantidad por partido, \%=porcentaje 
Rev.Ib.CC. Act. Fís. Dep. 2019; 8(1): 27-38

Tabla 4. Cantidad de victorias, empates y pérdidas de los equipos clasificados y no clasificados según el lugar donde se jugó el partido.

\begin{tabular}{lcccc}
\hline & \multicolumn{2}{c}{ Clasificados } & \multicolumn{2}{c}{ No clasificados } \\
\hline & Local & Visita & Local & Visita \\
\hline Victorias & 8 & 5 & 7 & 1 \\
Empates & 7 & 4 & 2 & 5 \\
Derrotas & 0 & 6 & 6 & 9 \\
Puntos & 31 & 19 & 23 & 8 \\
\hline
\end{tabular}

Los análisis de correlaciones entre las acciones ofensivas y el rendimiento, se observan en la tabla 5. La cantidad de remates a marco, la posesión de balón y pases realizados se correlacionaron positivamente con el rendimiento de los equipos, indicando, que los equipos que presentaron mejores resultados en estos indicadores tuvieron mejor rendimiento. Contrariamente, la cantidad de faltas se relacionó negativamente con el rendimiento, sugiriendo que los equipos que tuvieron mejor rendimiento cometieron menor cantidad de faltas.

En la tabla 6, se observa, que la acción defensiva que se correlacionó con el rendimiento de forma negativa fueron los goles recibidos, es decir, entre menor sea la cantidad de goles que reciba un equipo, mayor será su rendimiento.

Tabla 5. Correlaciones entre las acciones técnico-tácticas ofensivas y el rendimiento deportivo.

\begin{tabular}{lccccccccc}
\hline & $\mathbf{1}$ & $\mathbf{2}$ & $\mathbf{3}$ & $\mathbf{4}$ & $\mathbf{5}$ & $\mathbf{6}$ & $\mathbf{7}$ & $\mathbf{8}$ & $\mathbf{9}$ \\
\hline 1.Puntos & & & & & & & & & \\
2. Goles anotados (c/p) & 0,224 & & & & & & & \\
3. Remates a marco realizados (c/p) & 0,212 & $0,569^{*}$ & & & & & & \\
4. Tiros de esquina a favor (c/p) & 0,075 & $-0,073$ & 0,057 & & & & & \\
5. Fuera de juego recibidos (c/p) & 0,254 & $-0,086$ & 0,163 & 0,102 & & & & \\
6. Faltas recibidas (c/p) & $0,348^{*}$ & $-0,209$ & 0,070 & 0,073 & 0,146 & & & \\
7. Posesión balón (\%) & $0,435^{*}$ & 0,194 & 0,157 & 0,068 & 0,245 & 0,104 & & \\
8. Pases correctos (c/p) & $0,333^{*}$ & 0,174 & $0,325^{*}$ & 0,200 & $0,286^{*}$ & 0,012 & $0,679^{*}$ & \\
9. Efectividad Pases (\%) & 0,050 & 0,045 & 0,045 & 0,169 & 0,120 & $-0,100$ & 0,130 & $0,509^{*}$ & \\
\hline
\end{tabular}

* correlaciones $p<00,05$. Coeficientes de Spearman (rho)

Tabla 6. Correlaciones entre las acciones técnico-tácticas defensivas y el rendimiento deportivo.

\begin{tabular}{lcccccccc}
\hline & $\mathbf{1}$ & $\mathbf{2}$ & $\mathbf{3}$ & $\mathbf{4}$ & $\mathbf{5}$ & $\mathbf{6}$ & $\mathbf{7}$ & $\mathbf{8}$ \\
\hline 1.Puntos & & & & & & & & \\
2. Goles recibidos (c/p) & $-0,351^{*}$ & & & & & & \\
3. Remates a marco recibidos (c/p) & $-0,163$ & $0,578^{*}$ & & & & & \\
4. Tiros de esquina en contra (c/p) & 0,247 & $-0,185$ & 0,070 & & & & \\
5. Fuera de juego conseguidos (c/p) & $-0,170$ & $-0,113$ & 0,163 & 0,146 & & & & \\
6. Faltas cometidas (c/p) & 0,031 & $-0,085$ & 0,057 & 0,073 & 0,102 & & & \\
7. Tarjetas amarillas (c/p) & $-0,048$ & $0,363^{*}$ & $0,397^{*}$ & $0,313^{*}$ & 0,198 & $0,313^{*}$ & & \\
8. Tarjetas rojas (c/p) & 0,000 & $-0,114$ & $-0,157$ & $-0,273^{*}$ & $-0,146$ & 0,050 & $-0,012$ & \\
\hline
\end{tabular}

* correlaciones $p<0,05$. Coeficientes de Spearman (rho) 


\section{DISCUSIÓN}

Conocer cuáles fueron las acciones técnico-tácticas y factores contextuales que se relacionaron con el rendimiento de los equipos de CONCACAF durante la última fase clasificatoria para la Copa Mundial de Rusia 2018 fue el propósito de este estudio. Como este es uno de los primeros trabajos realizados que analiza los partidos eliminatorios hacia una copa mundial, los resultados obtenidos fueron comparados y contrastados con investigaciones previas que realizaron análisis similares a los de este estudio.

Los principales resultados encontrados en este estudio evidencian que acciones técnico-tácticas como los pases realizados, la posesión de balón y goles anotados fueron las variables que diferenciaron a los equipos que clasificaron con los que no clasificaron. Estos resultados se encuentran respaldados por previos estudios que han comparado el rendimiento técnico-táctico de equipos según el rendimiento alcanzado en los campeonatos. Estudios de las copas mundiales de 2002, 2006 y 2010 identificaron la posesión de balón, los goles y remates a marco como variables diferenciadoras entre equipos exitosos y no exitosos ${ }^{3,21}$. Además de estas variables, los pases realizados fueron también diferenciadores entre equipos que clasificaron y no a la ronda de octavos de final en el mundial de Brasil 2014,622,23. Igualmente, concuerda con los resultados encontrados en la liga española 2008-2009, al observarse que los equipos que se ubicaban en las primeras posiciones del ranking al finalizar la liga realizaron más goles, remates, asistencias y tuvieron porcentualmente más posesión de balón en comparación con equipos ubicados en posiciones inferiores del ranking ${ }^{15}$.

Este estudio reflejó que los equipos que clasificaron al mundial incurrieron mayor cantidad de veces en fueras de juego en comparación con los equipos que no clasificaron. Dos estudios en competiciones españolas, han reportado que los equipo que ganan se les ha sancionado en más veces fuera de juego en comparación con los equipos que empatan o pierden ${ }^{5,14}$. Se puede asociar que los equipos incurren mayormente en fueras de juego cuando intentan ir al ataque adelantando líneas e incorporando más jugadores a la ofensiva.

También, al tomar en cuenta los resultados obtenidos en los análisis de correlaciones, los mismos respaldan lo discutido anteriormente, sobre los indicadores que más se relacionan con el rendimiento; los cuales a su vez, son congruentes con la literatura. Por ejemplo, se ha observado en ligas brasileñas que una mayor cantidad de goles anotados y finalizaciones realizadas se correlacionó con una mayor cantidad de puntos conseguidos por los equipos durante la liga ${ }^{19}$. Asimismo, se ha identificado en trabajos de revisión que estas acciones técnicotácticas son comúnmente asociadas a resultados positivos y a un mejor rendimiento ${ }^{1,2}$.

Por su parte, al analizar los resultados obtenidos en este trabajo considerando los factores contextuales; los mismos se encuentran dentro de lo reportado en 
trabajos publicados previamente, en los cuales se ha visto que el hecho de jugar de local o de visita, así como el resultado que se obtuvo del partido son variables contextuales que influyen sobre los comportamientos técnico-tácticos y físicos de los jugadores y equipos $8,9,10,12$.

Considerando el resultados obtenido en el partido, los datos de este estudio son similares a los encontrados en la Primera División española en la temporada 2008-2009, donde se identificó que los equipos que ganaban realizaban mayor cantidad de remates totales, remates directos a marco, incurrían en más fueras de juego y realizaban más centros, mientras que los equipos que perdían presentaban mayor cantidad de centros recibidos, fueras de juego forzados y se les mostraba más tarjetas rojas ${ }^{14}$.

Los equipos que ganan en comparación con aquellos que empatan o pierden llegan con mayor frecuencia a la portería rival, aumentando sus posibilidades de rematar a marco y por ende de conseguir anotaciones ${ }^{15}$. Lo anterior, respaldado por investigaciones previas, que indican que los equipos que ganan manifiestan mejores comportamientos técnico-tácticos durante los partidos; permitiéndoles mantener un mejor control del juego, generando mayor cantidad de situaciones ofensivas sobre el equipo rival ${ }^{20,19}$. A su vez, los equipos que ganan muestran eficacia en acciones defensivas como intercepciones de balón, despejes y juegos aéreos ganados por partido, lo que contrarresta los ataques del equipo contrario ${ }^{3,11}$.

En cuanto a la influencia de la localía, este estudio encontró que los equipos que jugaban como local realizaron más goles, remates a marco, presentaban un mayor porcentaje de posesión de balón y menor cantidad de amonestaciones en comparación con los equipos visitantes, lo cual se encuentra dentro de los hallazgos de anteriores investigaciones, que indican que los equipos locales presenta valores favorables en estas variables ${ }^{14,13}$. Además, se ha determinado que los equipos locales limitan las acciones ofensivas del equipo rival, lo que probablemente incida en recibir menos anotaciones de local, lo cual les permite en muchos casos ganar el encuentro ${ }^{7}$.

Esta influencia de la localía sobre el resultado del partido puede verse en un estudio que dio seguimiento a 27 temporadas de la liga española y determino que los equipos consiguen mayor cantidad de victorias cuando juegan como locales en comparación cuando juegan de visita ${ }^{9}$. También, es importante abordar este tema desde la perspectiva psicológica, puesto que autores como Pollard $^{30}$, han expuesto que jugar de locales promueve un ambiente más favorable y positivo reuniendo una serie de condiciones como el apoyo de la afición y la familiaridad con el campo de juego que pueden repercutir en la mejora de las respuestas físicas, táctica y técnica tanto individuales como colectivas en acciones de ataque y defensa y que por tanto, es común observar mejores resultados para los equipos cuando disputan partidos de local en comparación a cuanto lo hacen de visita. 


\section{CONCLUSIONES}

Los resultados del estudio muestran que las acciones técnico-tácticas que más se relacionaron con el rendimiento fueron los goles anotados, remates a marco realizados, los pases y el porcentaje de posesión de balón, cuyos valores fueron más favorables para los equipos que clasificaron y que conseguían ganar los partidos. Asimismo, los resultados son congruentes con lo expresado en la literatura.

También, el hecho de jugar los partidos como locales representó ser una ventaja para los equipos, puesto que consiguieron más victorias en comparación a cuando jugaban de visita. Asimismo, se mostró que los equipos consiguieron mayor cantidad de anotaciones y realizaron más remates directos a marco.

\section{BIBLIOGRAFÍA}

1. Mackenzie R, Cushion C. Performance analysis in football: A critical review and implications for future research. Journal of Sports Sciencie. 2013;31:639-76.

2. Reina $A$, Hernández A. Revisión de indicadores de rendimiento en fútbol. Revista Iberoamericana de Ciencias de la Actividad Física y el Deporte. 2012;1(1):1-14.

3. Delgado JL, Domenech C, Guzmán JF. Mendez A. Offensive and defensive team performance: relation to successful and unsuccessful participation in the 2010 Soccer World Cup. Journal of Human Sport Exercise. 2013;8(4):894-904.

4. Liu H, Gómez MA, Goncalves B, Sampaio J. Technical performance and match-tomatch variation in elite football teams. Journal of Sports Sciencie. 2016;34:509-18.

5. Lago-Peñas C, Lago-Ballesteros J, Dellal A, Gómez M. Game-related statistics that discriminated winning, drawing and losing teams from the Spanish soccer league. Journal of Sports Science and Medicine. 2010;9:288-93.

6. Liu H, Gomez MÁ, Lago-Peñas C, Sampaio J. Match statistics related to winning in the group stage of 2014 Brazil FIFA World Cup. Journal of Sports Science. 2015;33(12):1205-13.

7. Almeida $\mathrm{CH}$, Ferreira AP, Volossovitch A. Effects of match location, match status and quality of opposition on regaining possession in UEFA Champions League. Journal of Human Kinetic. 2014;41:203-14.

8. Aquino R, Munhoz-Martins GH, Palucci-Vieira LH, Menezes RP. Influence of match location, quality of opponents, and match status on movement patterns in brazilian professional football players. Journal of Strength and Conditioning Research. 2017;31(8):2155-61.

9. Sánchez P, García-Calvo T, Leo F, Pollard R, Gómez M. An analysis of home advantage in the top two Spanish Professional Football leagues. Perceptual and Motor Skills. 2009;108(3):789-97.

10. Lago-Peñas C. Dellal A. Ball Possession Strategies in Elite Soccer According to the Evolution of the Match-Score: the Influence of Situational Variables. Journal of Human Kinetics. 2010;25:93-100.

11. Taylor JB, Mellalieu SD, James N, Shearer D. The influence of match location, qualify 
of opposition and match status on technical performance in professional association football. Journal of Sports Sciencie. 2008;26:885-95.

12. Castellano J, Blanco-Villaseñor A, Alvarez D. Contextual variables and time-motion analysis in soccer. International Journal of Sports Medicine. 2011;32(6):415-21.

13. Lago-Peñas C, Lago-Ballesteros J. (2011). Game location and team quality effects on performance profiles in professional soccer. Journal of Sports Science and Medicine. 2011;10:465-71.

14. Casáis L, Lago C, Lago J, Iglesias S, Gómez M. Indicadores de rendimiento competitivo que diferencian equipos ganadores y perdedores de la liga española. Revista de Preparación Física en el Fútbol. 2011;2:44-53.

15. Dellal A, Wong DP, Moalla W, Chamari K. Physical and technical activity of soccer players in the French First League - with special reference to their playing position. International Sport Medicine Journal. 2010;11(2):278-90.

16. Rampinini E, Impellizzeri FM, Castagna C, Coutts AJ, Wisloff U. Technical performance during soccer matches of the Italian Serie A league: Effect of fatigue and competitive level. Journal of Science and Medicine in Sport. 2009;12:227-33.

17. Russell M, Rees G, Kingsley M. Technical demands of soccer match-play in the English Championship. Journal of Strength and Conditioning Research. 2013;27(10):2869-73.

18. Silva SA, Silva CD, Paoli PB, Bottino AA, Marins JCB. Análise da correlação dos indicadores técnicos que determinam o desempenho das equipes no campeonato brasileiro de futebol. Revista Brasileira de Futebol. 2009;2(2):40-45.

19. Mahony LE, Wheeler KW, Lyons K. Analysis of factors determining invasion into attacking areas and the creation of goal scoring opportunities in the asian cup football competition. Asian Journal of Exercise \& Sports Science. 2012;9(1):53-66.

20. Vales Á, Blanco H, Areces A, Arce C. Perfiles de rendimiento de selecciones ganadoras y perdedoras en el Mundial de fútbol Sudáfrica 2010. Revista de Psicología del Deporte. 2015;24(1):111-18.

21. Castellano J, Casamichana D, Lago $\mathrm{C}$. The use of match statistics that discriminate between successful and unsuccessful soccer teams. Journal of Human Kinetics, 2012; 31:139-147.

22. Chmura P, Golinski D, Andrezejewski M, Mroczek D, Kawczynski, Rokita A, Chmura J. Endurance capacity and selected technical-tactical skills of soccer players participating in the group stage of the 2014 FIFA World Cup. Trends in Sport Sciences. 2015;3(21):153-60.

23. Kröl M, Konefal M, Chmura P, Andrzejewski M, Zajac T, Chmura J. Pass completion rate and match outcome at the World Cup in Brazil in 2014. Polish Journal of Sport and Tourism. 2017;24:30-34.

24. Confederación, Centroamericana, Norte y Caribe de Fútbol (CONCACAF). (2018). Calendario y resultados. Recuperado desde http://www.concacaf.com/es/premundialhombres/calendario-y-resultados-premundial-hombres\#tab_team=rounds

25. Rosenthal R. DiMatteo R. Meta-analysis: Recent developments in quantitative methods for literature reviews. Annual Review of Psychology. 2001;52:59-82.

26. Cohen J. Statistical power analysis for the behavioural sciences. Hillsdale: N.J.:L. Erlbaum Associates; 1998.

27. O'Donoghue P. Statistics for sport and exercise studies. An introduction. New York. USA: Taylor \& Francis Group; 2012. 
28. Rosenthal R. Rosnow RL. Essentials of behavioral research: Methods and data analysis. New York: McGraw-Hill; 1984.

29. Pollard R. Home advantage in football: A current review of an unsolved puzzle. Open Sport Sci J, 2008;1:12-14. 